\author{
Brief article
}

\title{
Differential involvement of left prefrontal cortex in inductive and deductive reasoning
}

\author{
Vinod Goel ${ }^{\mathrm{a}, \mathrm{b}, *}$, Raymond J. Dolan ${ }^{\mathrm{a}}$ \\ ${ }^{\mathrm{a}}$ Wellcome Department of Imaging Neuroscience, Institute of Neurology, UCL, \\ Queens Square, London WC1N 3BG, UK \\ ${ }^{\mathrm{b}}$ Department of Psychology, York University, Toronto, Ont., Canada M3J 1 P3
}

Received 5 November 2003; revised 4 February 2004; accepted 4 March 2004

\begin{abstract}
While inductive and deductive reasoning are considered distinct logical and psychological processes, little is known about their respective neural basis. To address this issue we scanned 16 subjects with fMRI, using an event-related design, while they engaged in inductive and deductive reasoning tasks. Both types of reasoning were characterized by activation of left lateral prefrontal and bilateral dorsal frontal, parietal, and occipital cortices. Neural responses unique to each type of reasoning determined from the Reasoning Type (deduction and induction) by Task (reasoning and baseline) interaction indicated greater involvement of left inferior frontal gyrus (BA 44) in deduction than induction, while left dorsolateral (BA 8/9) prefrontal gyrus showed greater activity during induction than deduction. This pattern suggests a dissociation within prefrontal cortex for deductive and inductive reasoning.

(C) 2004 Published by Elsevier B.V.
\end{abstract}

Keywords: Left prefrontal cortex; Inductive and deductive reasoning; fMRI studies

\section{Introduction}

Reasoning is the cognitive process of drawing inferences from given information. All arguments involve the claim that one or more propositions (the premises) provide some grounds for accepting another proposition (the conclusion). At first pass, arguments can be divided into deduction and induction. Deductive arguments can be evaluated for validity.

\footnotetext{
* Corresponding author. Address: Department of Psychology, York University, Toronto, Ont., Canada M3J 1P3. Tel.: +1-416-736-5121; fax: +1-416-736-5814.

E-mail address: vgoel@yorku.ca (V. Goel).

0022-2860/\$ - see front matter (C) 2004 Published by Elsevier B.V. doi:10.1016/j.cognition.2004.03.001
} 
Validity is a function of the relationship between premises and conclusion and involves the claim that the premises provide absolute grounds for accepting the conclusion (e.g. All men are mortal; Socrates is a man; $\therefore$ Socrates is mortal). Arguments where the premises provide only limited grounds for accepting the conclusion are broadly called inductive arguments (e.g. Socrates is a man; Socrates is mortal; $\therefore$ All men are mortal). Inductive arguments are never valid but can be evaluated for plausibility or reasonableness (as in the above example). Thus, while validity can be reduced to a function of the logical structure of sentences and arguments, plausibility is a function of sentence content and our knowledge of the world. It is usually a matter of knowing which properties generalize in the required manner and which do not.

Philosophically, induction and deduction constitute different categories of thought. They are also treated differently in the psychological literature (Garnham \& Oakhill, 1994; though see Johnson-Laird (1993) for an exception). We were interested in the functional anatomy of inductive and deductive reasoning, and in particular, the role of the prefrontal cortex in the two types of reasoning. Goel, Gold, Kapur and Houle (1997) addressed this question with a $\mathrm{O}^{15}$ PET study and reported that both induction and deduction activated a similar left frontal-temporal system and that induction differed from deduction in greater activation of medial dorsal prefrontal cortex (BA 8 and 9). However, the use of a block design necessitated by the temporal window of the PET technique made it difficult to disentangle set-related activity from activity that might be specific to a particular cognitive process of interest. Here, we address the same question with a more sensitive single-event fMRI design that provides us with a finer-grained analysis of the respective neuroanatomy of inductive and deductive reasoning, uncontaminated by issues of set or expectancy.

\section{Method}

\subsection{Subjects}

Sixteen right-handed normal subjects ( 8 males and 8 females), with a mean age of 27.5 years (SD 6.4) and mean education level of 17.8 years (SD 1.8), volunteered to participate in the study. All subjects gave informed consent and the study was approved by the Joint National Hospital for Neurology and Neurosurgery/Institute of Neurology Ethics Committee.

\subsubsection{Stimuli}

Fifty syllogisms (25 valid, 25 invalid), encompassing 24 different forms, ${ }^{1}$ fifty inductive arguments ( 25 plausible and 25 implausible as judged by a pilot subject), and 40 baseline trials were organized into a $2 \times 2$ factorial design (Fig. 1a). There was also a common rest condition consisting of 20 null events. The first factor was Reasoning Type, consisting of 2 levels, deduction (50 arguments, 20 baseline and 10 rest trials)

\footnotetext{
${ }^{1}$ The following forms of syllogisms (encoded as per Evans, Newstead, and Byrne (1993)) were utilized: AA1, AA2, AA4, AE1, AE4, AI1, AO2, EI1, EE3, EI1, EI2, EI3, EI4, E03, IA3, IA4, IE1, IE3, II1, IO1, OA3, OE1, OE2, OO1. Some valid forms also had invalid "counterparts".
} 


\begin{tabular}{|l|l|l|}
\hline & Deduction & Induction \\
\hline Reasoning & $\begin{array}{l}\text { All animals with 32 teeth are cats } \\
\text { No cats are dogs } \\
\text { No dogs have 32 teeth }\end{array}$ & $\begin{array}{l}\text { House cats have 32 teeth } \\
\text { Lions have 32 teeth } \\
\text { All felines have 32 teeth }\end{array}$ \\
\hline Baseline & $\begin{array}{l}\text { All animals with 32 teeth are cats } \\
\text { No cats are dogs } \\
\text { All zebras have stripes }\end{array}$ & $\begin{array}{l}\text { House cats have 32 teeth } \\
\text { Lions have 32 teeth } \\
\text { All elephants have trunks }\end{array}$ \\
\hline Rest & \multicolumn{2}{|l}{} \\
\hline
\end{tabular}

(a)

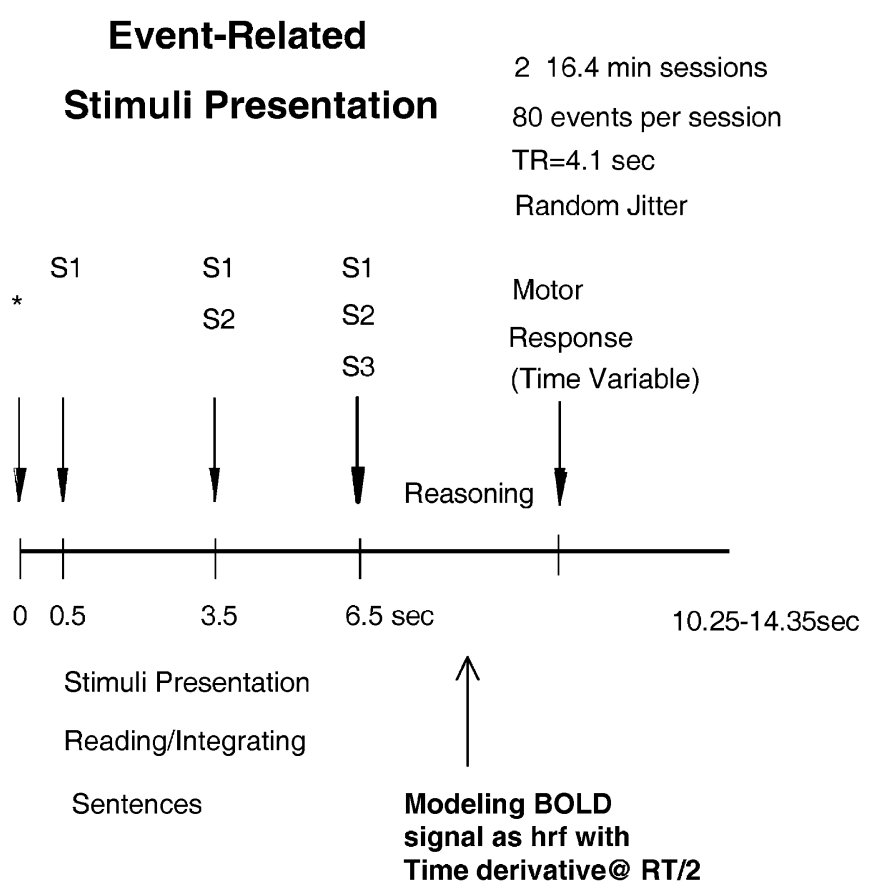

Fig. 1. (a) Overall design of study with sample stimuli. See text. (b) Stimuli presentation. See text.

and induction (50 arguments, 20 baseline and 10 rest trials) items. The two argument forms were matched for content. If there was a deductive argument about osteoporosis then there was also a matching inductive argument about osteoporosis (e.g. Deduction: No humans can get osteoporosis; Some humans are men; $\therefore$ Some men cannot get osteoporosis; and Induction: Osteoporosis is estrogen-related; Osteoporosis is common in women; $\therefore$ Men can also get osteoporosis).

The second factor was Task in which the first level was reasoning. Subjects were instructed to determine the validity of the syllogisms and plausibility of the inductive arguments. The deductive arguments were balanced for validity while the inductive arguments were balanced for plausibility. The second level (baseline condition) trials were generated by taking these arguments and switching around the 3rd sentence such that the three sentences did not constitute arguments. (e.g. No reptiles are hairy; Some elephants are hairy; 
$\therefore$ No pears are green and Some elephants are hairy; George is an elephant; $\therefore$ All pears are green). In the "rest" trials subjects simply viewed a series of Xs in the centre of a blank screen.

\subsubsection{Stimuli presentation}

To maintain consistent task instructions and mental set the deduction and induction trials were presented in separate blocks. Stimuli within the blocks were presented randomly in an event-related design (see Fig. 1b). The order of blocks was counterbalanced. The beginning of a trial was signaled by an "*". The sentences appeared on the screen one at a time with the first sentence appearing at $500 \mathrm{~ms}$, the second at $3500 \mathrm{~ms}$, and the last sentence at $6500 \mathrm{~ms}$. All sentences remained on the screen until the end of the trial. The length of trials varied from 10.25 to $14.35 \mathrm{~s}$, leaving subjects $3.75-7.85 \mathrm{~s}$ (after the presentation of the third sentence) to respond. In the rest trials sentences were replaced by a series of Xs calculated to occupy the same field of view.

\subsubsection{Task}

Subjects were required to reason in all but the rest trials. In the deduction session subjects were required to determine whether the given conclusion followed logically from the premises (i.e. whether the argument was valid). In the induction session subjects were required to determine whether the given conclusion was plausible given the premises. In baseline trials, where the first two sentences were related, subjects would need to begin to integrate the premises and construct a representation of the problem, ${ }^{2}$ but when the third, unrelated, sentence appeared they could immediately disengage from the task and respond 'no'. In trials where the three sentences constituted an argument (reasoning condition), subjects would need to continue with the integration of the conclusion (i.e. the reasoning component of the task) after the presentation of the third sentence. The difference between completing the reasoning task and disengaging after the presentation of the third sentence isolates the reasoning components of interest. ${ }^{3}$ In rest trials subjects simply viewed lines of Xs corresponding to the lines of text in the active trials.

In the active trials subjects provided a binary response by pressing one of two buttons on a keypad after the appearance of the last sentence (valid/invalid for deduction and plausible/implausible for induction). In rest trials subjects pressed any key after the presentation of the third line of Xs. Subjects were instructed to respond as quickly as possible and move to the next trial if the stimuli advanced before they could respond. Subjects reviewed example stimuli from each condition prior to being scanned to ensure that they understood the task.

\section{2. fMRI scanning technique}

A 2T Siemens VISION system (Siemens, Erlangen, Germany) was used to acquire T1 anatomical volume images $(1 \times 1 \times 1.5 \mathrm{~mm}$ voxels $)$ and $48 \mathrm{~T} 2 *$-weighted echoplanar

\footnotetext{
${ }^{2}$ Task difficulty and time limitations do not allow subjects the option of waiting until the presentation of the third sentence before deciding to begin integration of the first two sentences.

3 To further eliminate the neural activity associated with reading and encoding of the sentences we explicitly model them as events of no interest in all trials.
} 
images $(64 \times 643 \times 3 \mathrm{~mm}$ pixels, TE $40 \mathrm{~ms})$ sensitive to blood oxygenation level dependent (BOLD) contrast. Thin echoplanar images of $1.8 \mathrm{~mm}$ were acquired axially every $3 \mathrm{~mm}$, positioned to cover the whole brain. ${ }^{4}$ Data were recorded during a single acquisition period. A total of 494 volume images were acquired over two sessions (247 volumes per session) with a repetition time (TR) of $4.1 \mathrm{~s} /$ volume. The first six volumes in each session were discarded (leaving 241 volumes per session) to allow for $\mathrm{T} 1$ equilibration effects. Trials from all conditions were randomly presented in a single-event design. The mean trial time was $12,300 \mathrm{~ms}+/-2050 \mathrm{~ms}$ (TR) with a random jitter. Trials thus varied from 10.25 to $14.35 \mathrm{~s}$. There were 80 event presentations during a session for a total of 160 over the two sessions. Each session lasted $16.5 \mathrm{~min}$. The scanner was synchronized with the presentation of every trial in each session.

\section{3. fMRI data analysis}

Data were analyzed using Statistical Parametric Mapping (SPM 99) (Friston et al., 1995). All volumes were spatially realigned to the first volume (head movement was $<2 \mathrm{~mm}$ in all cases) and temporally realigned to the AC-PC slice, to account for different sampling times of different slices. A mean image created from the realigned volumes was coregistered with the structural $\mathrm{T} 1$ volume and the structural volumes spatially normalized to the Montreal Neurological Institute brain template (Evans, Collins et al., 1993) using nonlinear basis functions (Ashburner \& Friston, 1999). The derived spatial transformation was then applied to the realigned T2* volumes, which were finally spatially smoothed with a $12 \mathrm{~mm}$ FWHM isotropic Gaussian kernel (in order to make comparisons across subjects and to permit application of random field theory for corrected statistical inference (Worsley \& Friston, 1995)). The resulting time series across each voxel were high-pass filtered with a cut-off of $32 \mathrm{~s}$, using cosine functions to remove section-specific low frequency drifts in the BOLD signal. Global means were normalized by proportional scaling to a Grand Mean of 100, and the time series temporally smoothed with a canonical hemodynamic response function to swamp small temporal autocorrelations with a known filter.

All events were modeled in the design matrix, but the event of interest was the neural activity following presentation of a conclusion, coinciding with the reasoning component of the task. The BOLD signal corresponding to this event was modeled as a hemodynamic response function with time derivative at the midway point between the presentation of the third sentence and the motor response (on a trial by trial basis). The other events (i.e. the presentation of all three sentences and the motor response) were modeled as events of no interest (with a hemodynamic response function). Given known neural differences associated with correct and incorrect trials in deductive reasoning (Goel \& Dolan, 2003) only correct response deduction trials were incorporated into the design. As there is no measure of correct or incorrect response in induction, all inductive trials were incorporated in the analysis. Condition effects at each voxel were estimated according to the general

\footnotetext{
${ }^{4}$ The thin $1.8 \mathrm{~mm}$ slices, with $1.2 \mathrm{~mm}$ gap, and a relatively short echo time of $40 \mathrm{~ms}$ serve to minimize dropout and distortion (Deichmann, Josephs, Hutton, Corfield, \& Turner, 2002; Gorno-Tempini et al., 2002; Hutton et al., 2002).
} 
linear model and regionally specific effects compared using linear contrasts. Each contrast produced a statistical parametric map of the $t$-statistic for each voxel, which was subsequently transformed to a unit normal $Z$-distribution. We report all activations surviving a voxel-level intensity threshold of $p<0.05$ (corrected for multiple comparisons in a random effect model using False Discovery Rate) (Genovese, Lazar, $\&$ Nichols, 2002). An uncorrected threshold of $p<0.001$ was used for direct comparisons and interactions as they were inclusively masked by the main effect (approximating the joint probability of both effects).

\section{Results}

Behavioral scores indicated that subjects performed the task in the expected manner. Subjects took a mean of $3383 \mathrm{~ms}$ (SD 726) (after presentation of third sentence at $6500 \mathrm{~ms}$ ) to respond to the deductive arguments, significantly more than the $2552 \mathrm{~ms}$ (SD $605)$ required to respond to the inductive arguments. Both of these times were significantly greater than the reaction times for the baseline trials (1597 ms (SD 381) and $1538 \mathrm{~ms}$ (SD 298), respectively, for deduction and induction). Subjects had a mean correct score of $66.0 \%$ (SD 8.6) for deductive reasoning trials, significantly greater than chance $(t=7.45$, $p<0.001)$. In the inductive reasoning trials, there is of course no correct answer. ${ }^{5}$ Accuracy scores were significantly higher for deduction baseline trials (94.8\%, SD 14.9) and induction baseline trials (95.9\%, SD 9.0) than reasoning trials.

The FMRI results are summarized in Table 1. The main effect of reasoning [(deductive reasoning + inductive reasoning) - (deductive baseline + inductive baseline)] revealed activation in bilateral lingual gyri (BA 18), right inferior and middle occipital gyri (BA 18), left inferior temporal gyrus (BA 37), bilateral superior parietal lobule (BA 7), left inferior parietal lobule (BA 40), bilateral basal ganglia, right middle frontal gyrus (BA 6), medial frontal gyrus (BA 6), and left inferior frontal gyrus (BA 45).

Examining the simple effects of reasoning revealed that deductive reasoning (compared to baseline) was associated with activation in left cerebellum, bilateral inferior occipital gyrus (BA 18), left inferior temporal and occipital gyri (BA 37/19), left middle temporal gyrus (BA 39), right superior parietal lobule (BA 7), bilateral middle frontal gyrus (BA 6), bilateral putamen, and left inferior frontal gyrus (BA 44) (Fig. 2a). The simple effect of inductive reasoning (compared to baseline) activated a similar network consisting of right cerebellum, right lingual gyrus (BA 18), right middle occipital gyrus (BA 18), bilateral superior parietal lobule (BA 7), left inferior parietal lobule (BA 40), right middle frontal gyrus (BA 6), medial frontal gyrus (BA 6), putamen, and left middle frontal gyrus (BA 8/9/45) (Fig. 2b). Fig. 2c and d

\footnotetext{
${ }^{5}$ By definition, there are no correct/incorrect answers for the induction trials. However, we compared our subjects' responses in the induction trials to that of an age and education matched pilot subject who did the task outside the scanner. Compared to this individual subject, our scanned subjects performed the induction task at $64 \%$ accuracy, comparable to the $66 \%$ for the deduction trials. However, this is an arbitrary measure. Therefore we chose not to restrict the analysis of the fMRI data of the induction trials to "correct" responses.
} 
Table 1

\begin{tabular}{llll}
\hline Location (Brodmann area) & \multicolumn{2}{l}{ MNI coordinates } & $Z$ Z-score \\
\cline { 3 - 4 } & $X$ & $Y$ & $Z$
\end{tabular}

Main effect of reasoning

Rt. lingual gyrus (BA 18)

Lt. lingual gyrus (BA 18)

Rt. middle occipital gyrus (BA 18)

Rt. inferior occipital gyrus (BA 18)

Lt. inferior temporal gyrus (BA 37)

Lt. superior parietal lobule (BA 7)

Rt. superior parietal lobule (BA 7)

Lt. inferior parietal lobule (BA 40)

Rt. middle frontal gyrus (BA 6)

Rt. basal gangalia

Lt. basal gangalia

Medial frontal gyrus (BA 6)

Lt. inferior frontal gyrus (BA 45)

$\begin{array}{llll}12 & -93 & -9 & 3.95\end{array}$

Deductive reasoning - baseline

Lt. cerebellum

Rt. inferior occipital gyrus (BA 18)

Lt. inferior occipital gyrus (BA 18)

Lt. inferior temporal and occipital gyri (BA 37/19)

Lt. middle temporal gyrus (BA 39)

Rt. superior parietal lobule (BA 7)

Rt. middle frontal gyrus (BA 6)

Lt. middle frontal gyrus (BA 6)

Lt. middle frontal gyrus (BA 6)

Rt. putamen

Lt. putamen/caudate nucleus

Lt. inferior frontal gyrus (BA 44/45)

$\begin{array}{rrrr}12 & -93 & -9 & 3.95 \\ -6 & -90 & -9 & 3.52 \\ 33 & -81 & 12 & 4.72 \\ 36 & -93 & -3 & 4.57 \\ -48 & -66 & -12 & 4.43 \\ -24 & -57 & 60 & 4.48 \\ 21 & -60 & 60 & 4.45 \\ -45 & -36 & 54 & 4.20 \\ 36 & 0 & 60 & 5.36 \\ 9 & 6 & -12 & 3.59 \\ -15 & 18 & -9 & 3.70 \\ 0 & 15 & 66 & 3.79 \\ -54 & 24 & 12 & 4.35\end{array}$

Inductive reasoning - baseline

Rt. fusiform gyrus (BA 18)

Rt. lingual gyrus (BA 18)

Rt. middle occipital gyrus (BA 18)

Lt. superior parietal lobule (BA 7)

Rt. superior parietal lobule (BA 7)

Lt. inferior parietal lobule (BA 40)

$\begin{array}{rrrr}-45 & -54 & -36 & 4.43 \\ 36 & -90 & -6 & 4.45 \\ -33 & -96 & -3 & 3.93 \\ -54 & -69 & -9 & 4.08 \\ -48 & -63 & 27 & 4.22 \\ 24 & -60 & 57 & 3.57 \\ 36 & 3 & 63 & 4.81 \\ -27 & 12 & 63 & 3,64 \\ -42 & 6 & 45 & 3.68 \\ 12 & 6 & -12 & 3.60 \\ -15 & 3 & 3 & 3.46 \\ -54 & 21 & 6 & 4.79\end{array}$

Rt. middle frontal gyrus (BA 6)

Medial frontal gyrus (BA 6)

Lt. Putamen

Lt. middle frontal sulcus (BA 9/45)

Lt. middle frontal gyrus (BA 8/9)

$\begin{array}{rrrr}27 & -84 & -18 & 4.61 \\ 9 & -90 & -9 & 4.44 \\ 30 & -87 & 12 & 3.95 \\ -27 & -66 & 42 & 4.12 \\ 30 & -60 & 60 & 3.87 \\ -45 & -33 & 51 & 4.37 \\ 33 & 0 & 60 & 4.59 \\ -6 & 6 & 48 & 3.77 \\ -6 & 6 & -12 & 3.71 \\ -48 & 24 & 27 & 4.00 \\ -42 & 12 & 36 & 3.31\end{array}$

Interaction (modulating to deduction; masked inclusively by main effect of reasoning) Lt. inferior frontal gyrus (BA 44)

$-54 \quad 12$

Interaction (modulating to induction masked inclusively by main effect of reasoning)

Rt. superior occipital gyrus (BA 19)

$27 \quad-84$

$-36 \quad 21$

30 
Deduction vs. Baseline
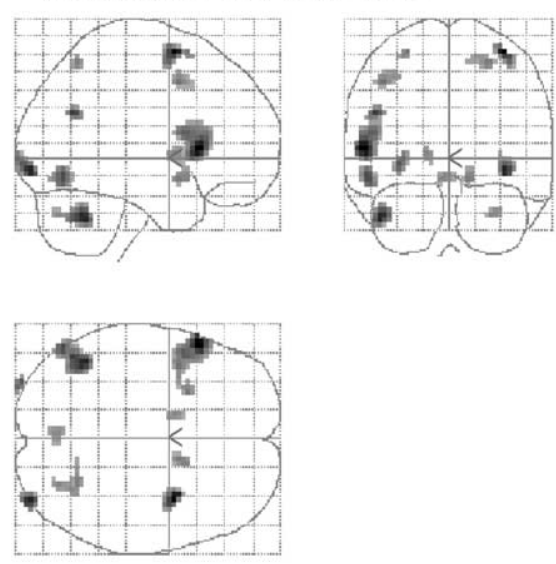

(a)

\section{Deduction vs. Rest}
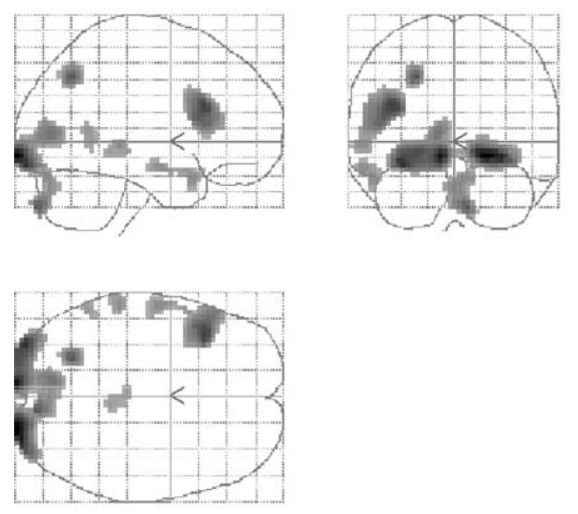

(c)
Induction vs. Baseline
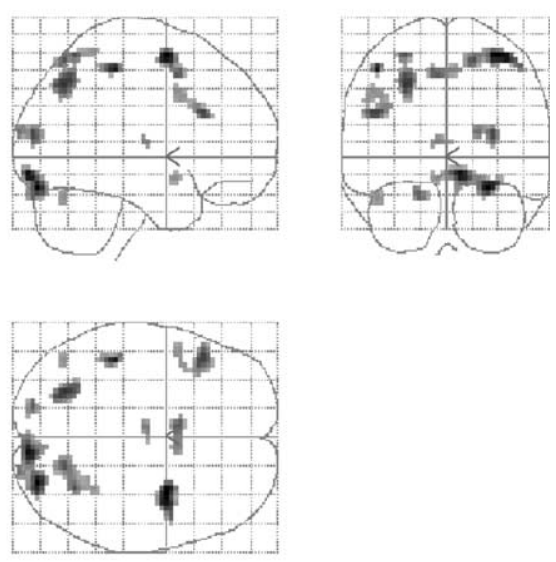

(b)
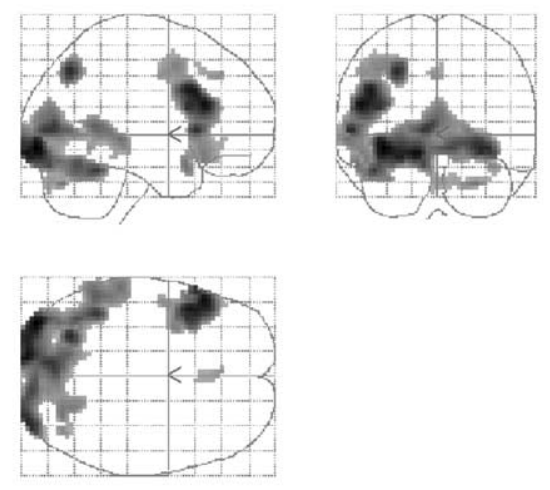

(d)

Fig. 2. Sagittal, coronal, and transverse views of a statistical parametric map (SPM) rendered into standard stereotactic space and projected onto a glass brain. (a) Simple effect of deduction (deductive reasoning baseline) (b) Simple effect of induction (inductive reasoning - baseline). (c) Deduction vs. rest $(p<0.0005$, uncorrected). (d) Induction vs. rest ( $p<0.0005$, uncorrected). See text.

show that the basic pattern of results remain unchanged even if deduction and induction are compared to the rest trials.

A Task (reasoning and baseline) by Reasoning Type (deduction and induction) interaction analysis revealed that deductive reasoning [(deductive reasoning deductive baseline) - (inductive reasoning - inductive baseline)] activated left inferior frontal gyrus (BA 44) over and above any effects seen during induction (Fig. $3 \mathrm{a}$ and $\mathrm{b}$ ). The reverse of this interaction [(inductive reasoning - inductive baseline) - (deductive reasoning - deductive baseline)] revealed enhanced activity in 


\section{Activations Unique to Deductive \& Inductive Reasoning Deductive Reasoning \\ [(Deduction - Base) - (Induction -Base)]}

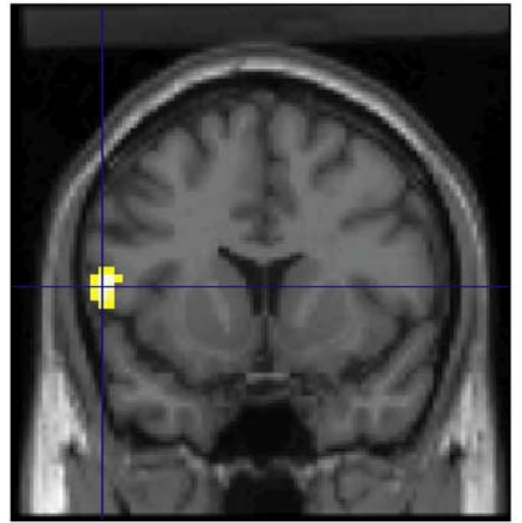

(a)

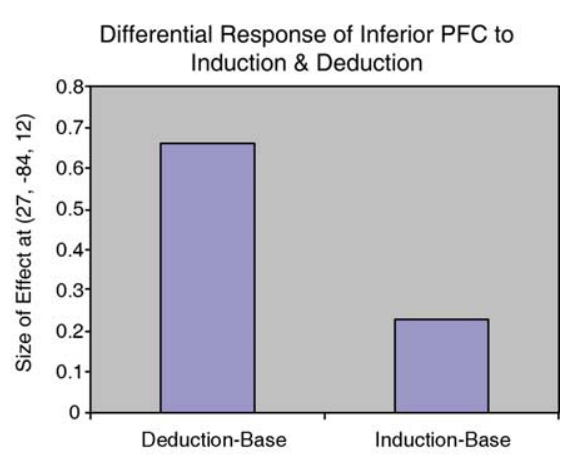

(b)

Inductive Reasoning [(Induction - Base) - (Deduction -Base)]

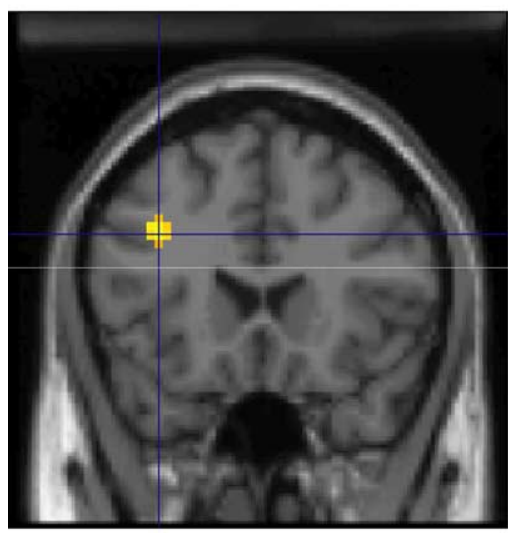

(c)

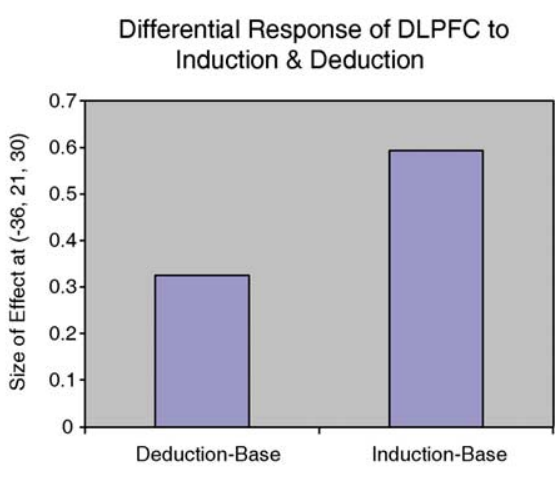

(d)

Fig. 3. A statistical parametric map (SPM) rendered into standard stereotactic space and superimposed on to transverse coronal sections of a magnetic resonance image (MRI) which is itself in standard space. (a) A task by reasoning type interaction analysis revealed that deductive reasoning [(deductive reasoning - deductive baseline) - (inductive reasoning - inductive baseline)], masked inclusively by the main effect of reasoning, activated left inferior frontal gyrus (BA 44) $(-54,12,12 ; Z=3.58)$. (b) Condition specific parameter (beta) estimates show that inferior prefrontal cortex is more functionally activated during deductive reasoning trials than during inductive reasoning trials. (c) The reverse interaction [(inductive reasoning - inductive baseline) (deductive reasoning - deductive baseline)], masked inclusively by the main effect of reasoning, revealed enhanced activity in left middle frontal gyrus (BA 9) $(-36,21,30 ; Z=3.08)$ during induction. (d) Condition specific parameter (beta) estimates show that dorsolateral prefrontal cortex is more functionally activated during inductive reasoning trials than during deductive reasoning trials. 
left middle frontal gyrus (BA 9) (Fig. 3c and d) and left superior occipital gyrus (BA 18) specific to induction.

\section{Discussion}

Our results indicate that both inductive and deductive reasoning engage a neural network comprising bilateral occipital, parietal, frontal dorsal, and left temporal, and lateral and dorsolateral frontal lobes. The results are consistent with previous neuroimaging and patient studies of reasoning. For example, the Goel et al. (1997) $\mathrm{O}^{15}$ PET study of inductive reasoning reported activation in a left frontal-temporal system. Gazzaniga and colleagues have administered simple inductive reasoning tasks to splitbrain patients and concluded that reasoning is a left hemisphere phenomenon. They offer the example that the left hemisphere will readily infer "boiling water" when presented with 'water' and 'pan' while the right hemisphere seems incapable of such inferences (Gazzaniga, 1985; Gazzaniga \& Smylie, 1984). Gazzaniga goes on to postulate a "left brain interpreter", a mechanism that continuously elaborates and interprets information presented to it and readily draws inferences (Gazzaniga, 1998).

A more recent case study (Varley \& Siegal, 2000) describes a patient with a lesion encompassing inferior lateral prefrontal cortex and temporal lobe regions. According to the patient description, anatomical scan, and "executive functions" task scores provided, the dorsolateral and medial aspects of the prefrontal cortex were intact. The basic finding was that while the linguistic/grammatical ability of the patient was severely impaired, some "theory of mind" (ToM) reasoning ability was spared. The theory of mind tasks administered all involved inductive reasoning. The fact that this patient performed an induction (more specifically ToM induction) task, is consistent with our finding that induction involves more dorsolateral aspects of the left prefrontal cortex, and medial prefrontal cortex. It is also consistent with claims in the literature of medial prefrontal cortex involvement in ToM reasoning (Frith \& Frith, 2003; Goel, Grafman, Sadato, \& Hallet, 1995).

A series of $\mathrm{O}^{15} \mathrm{PET}$ and fMRI studies on deductive reasoning have revealed activation in bilateral occipital, parietal and left lateral temporal and prefrontal cortex (Acuna, Eliassen, Donoghue, \& Sanes, 2002; Christoff et al., 2001; Goel, Buchel, Frith, \& Dolan, 2000; Goel \& Dolan, 2000, 2001, 2003; Goel et al., 1995, 1997; Goel, Gold, Kapur, \& Houle, 1998; Houde et al., 2000; Knauff, Mulack, Kassubek, Salih, \& Greenlee, 2002; Kroger et al., 2002). Patient studies of deduction report similar results. For example, Read (1981) tested temporal lobectomy patients on three term relational problems with semantic content and reported that $\mathrm{LH}$ patient performance was more impaired than $\mathrm{RH}$ patient performance. Caramazza, Gordon, Zurif, and DeLuca (1976) administered two-term problems such as the following: "Mike is taller than George. who is taller?" They reported that left hemisphere patients were impaired in all forms of the problem but-consistent with imaging data (Goel et al., 2000; Goel \& Dolan, 2003)—right hemisphere patients were only impaired when the form of the question was incongruent with the premise (e.g. who is shorter?). Similarly, Langdon and Warrington (2000) used matched verbal 
and spatial reasoning tasks and found that only left hemisphere patients failed the verbal section, while both left and right hemisphere patients failed the spatial sections.

Finally, Goel, Shuren, Sheesley and Grafman (2004) tested patients with focal frontal lobe lesions on the Wason Card Selection Task (Wason \& Shapiro, 1971), while manipulating social knowledge. The Wason Card Selection Task is generally regarded as a deductive reasoning task, but some researchers consider it to be an inductive reasoning task (Garnham \& Oakhill, 1994). Patients and controls performed equivalently on the arbitrary rule condition. Normal controls (along with patients with right frontal lobe lesions) displayed the expected improved performance in the social knowledge conditions. Patients with left frontal lobe lesions selectively failed to show this facilitation in performance, suggesting an asymmetry in frontal lobe $(L>R)$ involvement in reasoning.

The only study in the literature at variance with our present findings is a $\mathrm{O}^{15} \mathrm{PET}$ study (Parsons \& Osherson, 2001) which reported that deduction activates right hemisphere systems while induction (probabilistic reasoning) activates mostly left hemisphere systems. However, the findings in this study are at variance with the majority of imaging studies of deductive reasoning (Acuna et al., 2002; Goel et al., 1997, 1998, 2000; Goel \& Dolan, 2001, 2003; Knauff et al., 2002) as well as the patient data cited above.

The apparent contradiction between studies may be explained in terms of differences in design and/or stimuli differences. Parsons and Osherson (2001) were limited to a block design and chose to use identical arguments in the induction and deduction conditions. The use of a block design precludes the possibility of differentiating the reading of the stimuli sentences from the reasoning task and the isolating of correct from incorrect trials. The use of identical stimuli in the induction and deduction conditions has merit, but comes at a cost. One can only use invalid deductive arguments, thus introducing an asymmetry into the design. This may result in differences in neural response. ${ }^{6}$ In terms of stimuli, Parsons and Osherson (2001) used complex conditional statements while our (deductive) arguments consist of categorical syllogisms. The former incorporate logical operators while the latter incorporate quantification and negation relations. It is also possible there are neural differences in how statements involving logical operators and quantification and negation relations are processed.

The main interest in the present study are the findings from the Task by Reasoning Type interaction analyses that show greater left inferior frontal gyrus (BA 44) (Broca's Area) activation for deduction than induction. Conversely, the left dorsolateral (BA 9) prefrontal cortex (along with right superior occipital gyrus (BA 19)) show greater increases for induction. This result is reinforced by the simple effects analyses, where deduction minus baseline activates left inferior frontal gyrus and induction minus baseline activates more dorsal regions of left prefrontal cortex. There are several possible reasons for the greater involvement of Broca's Area (BA 44) in deduction than induction. Broca's Area is part of the phonological loop of working memory, and deductive reasoning has greater working memory requirements than inductive reasoning (Gilhooly, Logie, Wetherick, \& Wynn, 1993). Broca's Area is also involved in syntactic processing, and as mentioned in the Introduction, the validity of deductive arguments is a function of logical form, which in

\footnotetext{
${ }^{6}$ Indeed a direct comparison (unmasked) of invalid with valid trials reveals activation in right dorsolateral PFC (39, 42, 27; $Z=3.87)$, though it does not survive correction.
} 
turn is encoded in syntactic structure. Thus enhanced activity in Broca's Area during deduction may be a function of greater engagement of syntactical processing and greater working memory requirements.

Inductive reasoning on the other hand, is sensitive to background knowledge rather than logical form. The increased activity in dorsolateral prefrontal cortex may thus be due to the use of world knowledge in the generation and evaluation of hypotheses (Grafman, 2002) which is the basis of inductive inference. Consistent with this dissociation between lateral and dorsolateral prefrontal cortex, lesion studies typically implicate the dorsolateral prefrontal cortex in everyday reasoning deficits (Shallice \& Burgess, 1991; Stuss \& Alexander, 2000), while neuroimaging studies have consistently activated lateral prefrontal cortex in logical (deductive) reasoning tasks (Goel et al., 1997, 1998, 2000; Goel \& Dolan, 2003). Given that most everyday reasoning is inductive, this puzzle may be explained by the different roles for lateral and dorsolateral prefrontal cortex in reasoning suggested by our results.

In summary, our findings provide additional evidence for left hemisphere dominance in human reasoning and more accurately identify brain regions unique to inductive and deductive reasoning. Contrary to common expectations in the hemispheric asymmetry literature (Springer \& Deutsch, 1998) - but consistent with existent lesion datadeduction and induction do not activate left and right prefrontal cortex, respectively. Both forms of reasoning involve left prefrontal cortex. Consistent with its greater requirements for syntactic processing and working memory, deduction is characterized by increased activation in Broca's Area while induction involves greater activation in left dorsolateral prefrontal cortex, consistent with its need to access and evaluate world knowledge.

\section{References}

Acuna, B. D., Eliassen, J. C., Donoghue, J. P., \& Sanes, J. N. (2002). Frontal and parietal lobe activation during transitive inference in humans. Cerebral Cortex, 12(12), 1312-1321.

Ashburner, J., \& Friston, K. J. (1999). Nonlinear spatial normalization using basis functions. Human Brain Mapping, 7(4), 254-266.

Caramazza, A., Gordon, J., Zurif, E. B., \& DeLuca, D. (1976). Right-hemispheric damage and verbal problem solving behavior. Brain and Language, 3(1), 41-46.

Christoff, K., Prabhakaran, V., Dorfman, J., Zhao, Z., Kroger, J. K., Holyoak, K. J., \& Gabrieli, J. D. (2001). Rostrolateral prefrontal cortex involvement in relational integration during reasoning. NeuroImage, 14(5), 1136-1149.

Deichmann, R., Josephs, O., Hutton, C., Corfield, D. R., \& Turner, R. (2002). Compensation of susceptibilityinduced BOLD sensitivity losses in echo-planar fMRI imaging. NeuroImage, 15(1), 120-135.

Evans, A. C., Collins, D. L., Mills, S. R., Brown, E. D., Kelly, R. L., \& Peters, T. M. (1993). 3D statistical neuroanatomical models from 305 MRI volumes. Proceedings of the IEEE-Nuclear Science Symposium and Medical Imaging Conference, 1813-1817.

Evans, J. S. B. T., Newstead, S. E., \& Byrne, R. M. J. (1993). Human reasoning: The psychology of deduction. Hillsdale, NJ: Lawrence Erlbaum.

Frith, U., \& Frith, C. D. (2003). Development and neurophysiology of mentalizing. Philosophical Transactions of the Royal Society of London. Series B: Biological Sciences, 358(1431), 459-473.

Friston, K. J., Holmes, A. P., Worsley, K. J., Poline, J. B., Frith, C. D., \& Frackowiak, R. S. J. (1995). Statistical parametric maps in functional imaging: A general approach. Human Brain Mapping, 2, 189-210.

Garnham, A., \& Oakhill, J. (1994). Thinking and reasoning. Oxford: Blackwell. 
Gazzaniga, M. S. (1985). The social brain. New York: Basic Books.

Gazzaniga, M. S. (1998). The mind's past. Berkeley, CA: University of California Press.

Gazzaniga, M. S., \& Smylie, C. S. (1984). Dissociation of language and cognition. Brain, 107, 145-153.

Genovese, C. R., Lazar, N. A., \& Nichols, T. (2002). Thresholding of statistical maps in functional neuroimaging using the false discovery rate. NeuroImage, 15(4), 870-878.

Gilhooly, K. J., Logie, R. H., Wetherick, N. E., \& Wynn, V. (1993). Working memory and strategies in syllogistic-reasoning tasks. Memory and Cognition, 21, 115-124.

Goel, V., Buchel, C., Frith, C., \& Dolan, R. J. (2000). Dissociation of mechanisms underlying syllogistic reasoning. NeuroImage, 12(5), 504-514.

Goel, V., \& Dolan, R. J. (2000). Anatomical segregation of component processes in an inductive inference task. Journal of Cognitive Neuroscience, 12(1), 1-10.

Goel, V., \& Dolan, R. J. (2001). Functional neuroanatomy of three-term relational reasoning. Neuropsychologia, 39(9), 901-909.

Goel, V., \& Dolan, R. J. (2003). Explaining modulation of reasoning by belief. Cognition, 87(1), B11-B22.

Goel, V., Gold, B., Kapur, S., \& Houle, S. (1997). The seats of reason: A localization study of deductive and inductive reasoning using PET (O15) blood flow technique. NeuroReport, 8(5), 1305-1310.

Goel, V., Gold, B., Kapur, S., \& Houle, S. (1998). Neuroanatomical correlates of human reasoning. Journal of Cognitive Neuroscience, 10(3), 293-302.

Goel, V., Grafman, J., Sadato, N., \& Hallet, M. (1995). Modelling other minds. NeuroReport, 6(13), 1741-1746

Goel, V., Shuren, J., Sheesley, L., \& Grafman, J. (2004). Asymmetrical involvement of frontal lobes in social reasoning. Brain, $127(3), 1-8$.

Gorno-Tempini, M. L., Hutton, C., Josephs, O., Deichmann, R., Price, C., \& Turner, R. (2002). Echo time dependence of BOLD contrast and susceptibility artifacts. NeuroImage, 15(1), 136-142.

Grafman, J. (2002). The structured event complex and the human prefrontal cortex. In D. T. Stuss, \& R. T. Knight (Eds.), The frontal lobes (pp. 292-310). New York: Oxford University Press.

Houde, O., Zago, L., Mellet, E., Moutier, S., Pineau, A., Mazoyer, B., \& Tzourio-Mazoyer, N. (2000). Shifting from the perceptual brain to the logical brain: The neural impact of cognitive inhibition training. Journal of Cognitive Neuroscience, 12(5), 721-728.

Hutton, C., Bork, A., Josephs, O., Deichmann, R., Ashburner, J., \& Turner, R. (2002). Image distortion correction in fMRI: A quantitative evaluation. Neurolmage, 16(1), 217-240.

Johnson-Laird, P. N. (1993). Human and machine thinking. Hillsdale, NJ: Erlbaum.

Knauff, M., Mulack, T., Kassubek, J., Salih, H. R., \& Greenlee, M. W. (2002). Spatial imagery in deductive reasoning: A functional MRI study. Brain Research. Cognitive Brain Research, 13(2), 203-212.

Kroger, J. K., Sabb, F. W., Fales, C. L., Bookheimer, S. Y., Cohen, M. S., \& Holyoak, K. J. (2002). Recruitment of anterior dorsolateral prefrontal cortex in human reasoning: A parametric study of relational complexity. Cerebral Cortex, 12(5), 477-485.

Langdon, D., \& Warrington, E. K. (2000). The role of the left hemisphere in verbal and spatial reasoning tasks. Cortex, 36(5), 691-702.

Parsons, L. M., \& Osherson, D. (2001). New evidence for distinct right and left brain systems for deductive versus probabilistic reasoning. Cerebral Cortex, 11(10), 954-965.

Read, D. E. (1981). Solving deductive-reasoning problems after unilateral temporal lobectomy. Brain and Language, 12, 116-127.

Shallice, T., \& Burgess, P. (1991). Higher-order cognitive impairments and frontal lobe lesions in man. In H. S Levin, H. M. Eisenberg, \& A. L. Benton (Eds.), Frontal lobe function and dysfunction. Oxford: Oxford University Press.

Springer, S. P., \& Deutsch, G. (1998). Left brain, right brain (5th ed). San Francisco, CA: Freeman.

Stuss, D. T., \& Alexander, M. P. (2000). Executive functions and the frontal lobes: A conceptual view. Psychological Research, 63(3-4), 289-298.

Varley, R., \& Siegal, M. (2000). Evidence for cognition without grammar from causal reasoning and theory of mind in an agrammatic aphasic patient. Current Biology, 10(12), 723-726.

Wason, P. C., \& Shapiro, D. A. (1971). Natural and contrived experience in a reasoning problem. Quarterly Journal of Experimental Psychology, 23, 63-71.

Worsley, K. J., \& Friston, K. J. (1995). Analysis of fMRI time-series revisited-Again. NeuroImage, 2, 173 - 181 